\title{
Extraintestinal Manifestations of Inflammatory Bowel Disease in Middle Eastern Patients
}

\author{
Heba Adam ${ }^{1}$, Maryam Alqassas ${ }^{1}$, Omar I. Saadah ${ }^{2,3}$, Mahmoud Mosli ${ }^{1,3, *}$ \\ ${ }^{1}$ Department of Medicine, Faculty of Medicine, King Abdulaziz University, Jeddah, Saudi Arabia \\ ${ }^{2}$ Department of Pediatrics, Faculty of Medicine, King Abdulaziz University, Jeddah, Saudi Arabia \\ ${ }^{3}$ Inflammatory Bowel Disease Research Group, King Abdulaziz University, Jeddah, Saudi Arabia
}

\section{ARTICLE INFO}

\section{Article History}

Received 24 August 2019

Accepted 01 March 2020

\section{Keywords}

Inflammatory bowel disease extraintestinal manifestations incidence predictors

\begin{abstract}
Background and Aims: The Inflammatory Bowel Diseases (IBDs), Crohn's Disease (CD) and Ulcerative Colitis (UC), are gastrointestinal autoimmune disorders with many Extraintestinal Manifestations (EIMs). Previously reported incidences of EIMs in IBD patients have ranged from $10 \%$ to $50 \%$. The large variation in occurrence of EIMs has been linked to genetic predisposition. Correlations between individual EIMs are unclear. Therefore, we aim to estimate the incidence of EIMs in a Middle Eastern cohort of patients with IBD and examine possible relationships with EIMs.
\end{abstract}

Patients and Methods: We conducted a retrospective study involving all patients included in the King Abdulaziz University IBD information system registry between 2013 and 2018. Data on demographics, disease characteristics, and EIMs were extracted and analyzed using descriptive statistics: the standard Student's $t$-test and chi-squared test. Logistic regression analysis was used to examine associations using STATA software version 11.2 (StataCorp, TX, USA).

Results: We reviewed the electronic medical files of 284 patients with confirmed IBD, of which 158 (55.6\%) were females, the mean age was $27.8( \pm 15)$ years; $146(51.4 \%)$ patients had CD and $138(48.6 \%)$ UC. The overall incidence risk of EIMs was 138 $(52.3 \%)$ over a mean duration of follow up of $7.3( \pm 3.9)$ years. The most common EIM was arthritis (33\%), followed by aphthous ulcers (16\%). Pyoderma gangrenosum occurred in $8 \%$ of patients and appeared to be specific for CD patients $(p=0.002)$, whereas Primary Sclerosing Cholangitis (PSC) was more specific for UC $(p=0.001)$. Certain EIMs appeared to occur together such as arthritis with PSC $(p=0.001)$. Regression analysis identified disease type (in favor of UC; odds ratio $=0.50, p=0.03$ ) and age at the time of diagnosis (odds ratio $=1.04, p=0.001$ ) as the only significant predictors of EIMs.

Conclusion: Our results demonstrate that more than half of IBD patients have at least one EIM. Contrary to what has often been reported, we found that EIMs occur more commonly in UC than CD. A multidisciplinary assessment is recommended as part of IBD management to improve overall health outcomes.

(C) 2020 Atlantis Press International B.V.

This is an open access article distributed under the CC BY-NC 4.0 license (http://creativecommons.org/licenses/by-nc/4.0/)

\section{INTRODUCTION}

Inflammatory Bowel Diseases (IBDs) are multifactorial diseases that cause continuous inflammation of the gastrointestinal tract [1]. Although the prevalence and incidence of IBD are highest in Western populations, Asia and the Middle East have witnessed a large increase in prevalence, with estimates of $4.9-168.3$ per 100,000 persons with Ulcerative Colitis (UC) cases as compared with an increase of 37.5-248.6 per 100,000 persons in North America. Crohn's Disease (CD) has showed a variation in incidence globally, with reports of an increase of 0.88-67.9 per 100,000 persons in Asia and the Middle East compared with a much higher prevalence in North America, from 16.7 to 318.5 per 100,000 persons [2]. IBD has become an increasing concern due to its relapsing gastrointestinal manifestations and its association with other systems. Disease-related morbidity can significantly affect quality

"Corresponding author.Email: mmosli@kau.edu.sa

Data availability statement: The data that support the findings of this study are available from the corresponding author (MM), upon reasonable request. of life and work productivity, and may lead to increased health expenditure [3].

Extraintestinal Manifestations (EIMs) of IBD, which involve the joints, eyes, skin, and biliary tract, have a marked impact on the morbidity and mortality of IBD, with overall occurrence frequencies of $6-47 \%$ [4-11]. However, several studies conducted in Asia have found that EIMs are less prevalent among IBD patients when compared with those in Western countries [12,13]. A systematic review found that the most common of these manifestations are inflammatory arthropathies, with a prevalence of $10-35 \%$ [14]. Other common EIMs include oral and ocular lesions, which reportedly occur in $4-20 \%$ and $2-29 \%$ of IBD patients, respectively. The skin may also be involved in the form of Erythema Nodosum (EN) in $2-20 \%$ of cases and Pyoderma Gangrenosum (PG) in $0.5-2 \%$ of cases [14,15]. A group of manifestations may occur secondary to metabolic and anatomical features of the disease itself, such as osteopenia and osteoporosis, anemia, cholelithiasis, and nephrolithiasis [11]. One-fifth of IBD patients are affected with more than one EIM at a time, as shown by a study conducted in 2015 [16]. Researchers in Hungary have found that EIMs are more common 
in CD than in UC patients, excluding ocular manifestations and Primary Sclerosing Cholangitis (PSC). Gender differences and EIMs that vary according to the phenotype of the disease have also been found [17]. Most of these manifestations appear after IBD has been diagnosed; however, they have been shown in one study to appear before IBD diagnosis in $25.8 \%$ of cases [18].

Studies conducted in Saudi Arabia have found that the most common EIMs found in UC patients are osteopenia, followed by EN, and then arthritis, with a prevalence of $30.5 \%, 23.8 \%$, and $17.5 \%$, respectively [19]. However, only $5 \%$ of CD patients have dermatological manifestations, $15.5 \%$ have arthritis or arthralgia, and 2.3\% have deep vein thrombosis (DVT) [20]. A study in Riyadh showed that $32 \%$ of pediatric IBD patients have EIMs, most of them have indeterminate colitis [21].

Despite many studies that have investigated the prevalence of EIMs in IBD populations across the world, both cohort and crosssectional, few have been conducted in the Middle East. Therefore, we aim to investigate the incidence of EIMs and examine possible relationships between EIMs and key demographic and clinical factors in IBD patients living in the Middle East.

\section{PATIENTS AND METHODS}

We conducted a retrospective study involving all patients diagnosed with IBD between 2013 and 2018 using the International Classification of Diseases coding 10th Revision (ICD-10). We used the IBD Information System (IBDIS) database at King Abdulaziz University to identify patients with IBD. Patients with confirmed IBD according to conventional clinical, radiographic, endoscopic, and histological criteria were included, irrespective of age. Demographic, clinical, and endoscopic findings were ascertained directly from the IBDIS. Clinical, radiological, and laboratory findings were reviewed to confirm the presence or absence of EIMs.

\subsection{Outcomes and Definitions}

Examining the incidence of EIMs (overall and individual) was considered the primary outcome of this study. EIMs were defined as any manifestation linked with IBD, which either occurred during the course of disease or before IBD diagnosis. These included: arthritis, aphthous ulcers, scleritis/episcleritis, EN, PG, PSC, and Venous Thromboembolism (VTE).

\subsection{Statistical Analysis}

Summary statistics were used to describe baseline characteristics of the studied population. We used means and Standard Deviations (SDs) to summarize continuous variables and frequencies for categorical variables. Comparisons between means were carried out using the Student's $t$-test for normally distributed variables and the Wilcoxon signed-ranks test for non-normally distributed variables. The chi-squared test and ANOVA test were used to compare frequencies, where appropriate. The incidence risk of EIMs was calculated using the standard formula. Simple and multiple logistic regression analysis were used to study associations between EIMs and disease characteristics. Odds Ratios (ORs) were used to express associations. Precision of point estimates were determined by 95\% Confidence Intervals (CIs) and a $P$-value of $<0.05$ was considered statistically significant. STATA 11.2 (StataCorp, TX, USA) was used to perform the analysis.

\subsection{Ethical Considerations}

King Abdulaziz University ethics committee provided approval before study initiation.

\section{RESULTS}

\subsection{Baseline Characteristics}

The electronic files of 284 patients were reviewed. Twenty individuals were excluded owing to missing data. The majority of patients were females $(n=158,55.6 \%)$ and their average age was 27.8 years (range $=8-73, \mathrm{SD}=15.5$ ), all of which were of Middle Eastern descent. CD was more common than UC ( $n=146,51.4 \%$ vs. $n=$ $138,48.6 \%)$. Table 1 summarizes all baseline characteristics.

\subsection{Extraintestinal Manifestations}

Of the 284 patient files reviewed, 138 (48.6\%) reported having experienced EIMs. Overall, there was no difference in incidence between UC and CD ( $n=70$ vs. $n=68, p=0.69)$. The most common EIM was arthritis $(n=88,33.3 \%)$, which occurred more in UC compared with CD ( $n=55$ vs. $n=33, p=0.02$ ), followed by aphthous oral ulcers $(n=42,15.9 \%)$. PSC and VTE occurred more in UC patients compared with CD patients ( $n=17$ vs. $n=1, p=$ 0.001 , and $n=8$ vs. $n=2, p=0.07$, respectively). PG was encountered more frequently in CD patients than UC patients $(n=17$ vs. $n=4, p=0.002$ ). Table 2 summarizes the frequency of each EIM.

\subsection{Correlations between EIMs According to Simple Logistic Regression}

According to simple logistic regression analysis, EIMs were not predicted by the type of IBD (OR $=0.91, p=0.56)$. Certain EIMs were found to be more likely to occur together, such as arthritis

Table 1 Baseline characteristics of 284 patients with inflammatory bowel disease

\begin{tabular}{lc}
\hline Characteristics & Value \\
\hline Age in years $( \pm \mathrm{SD})$ & $27.8( \pm 15.5)$ \\
Age at diagnosis in years $( \pm \mathrm{SD})$ & $22.1( \pm 14.5)$ \\
Duration of disease in years $( \pm \mathrm{SD})$ & $7.3( \pm 3.9)$ \\
Gender & \\
Male $(\%)$ & $126(44.4)$ \\
Female $(\%)$ & $158(55.6)$ \\
Clinical diagnosis & \\
Ulcerative colitis $(\%)$ & $138(48.6)$ \\
Crohn's disease $(\%)$ & $146(51.4)$ \\
Smokers $(\%)$ & $39(16.7)$ \\
Family history of inflammatory bowel disease $(\%)$ & $38(14.5)$ \\
\hline
\end{tabular}


Table 2 Incidence risk of extra intestinal manifestations in Inflammatory Bowel Disease in a cohort of 264 patients

\begin{tabular}{|c|c|c|c|c|}
\hline Extraintestinal manifestation & $\begin{array}{l}\text { Ulcerative colitis } \\
(n=137)(\%)\end{array}$ & $\begin{array}{l}\text { Crohn's disease } \\
(n=127)(\%)\end{array}$ & $\begin{array}{l}\text { Overall incidence risk } \\
\quad(n=264)(\%)\end{array}$ & $p$-value \\
\hline All extraintestinal manifestations & $70(26.5)$ & $68(25.8)$ & $138(52.3)$ & 0.700 \\
\hline Arthritis & $55(20.8)$ & $33(12.5)$ & $88(33.3)$ & 0.020 \\
\hline Aphthous ulcers & $21(8.0)$ & $21(8.0)$ & $42(15.9)$ & 0.790 \\
\hline Scleritis/episcleritis & $13(5.0)$ & $16(6.0)$ & $29(11)$ & 0.420 \\
\hline Erythema nodosum & $14(5.3)$ & $12(4.5)$ & $26(9.85)$ & 0.830 \\
\hline Pyoderma gangrenosum & $4(1.5)$ & $17(6.4)$ & $21(7.95)$ & 0.002 \\
\hline Primary sclerosing cholangitis & $17(6.4)$ & $1(0.4)$ & $18(6.8)$ & 0.001 \\
\hline Venous thromboembolism & $8(3.0)$ & $2(0.8)$ & $10(3.8)$ & 0.070 \\
\hline
\end{tabular}

Table 3 Odds ratios correlating extraintestinal manifestations of inflammatory bowel disease

\begin{tabular}{|c|c|c|c|c|c|c|c|}
\hline $\begin{array}{l}\text { Extraintestinal } \\
\text { manifestations }\end{array}$ & $\begin{array}{l}\text { Primary } \\
\text { sclerosing } \\
\text { cholangitis }\end{array}$ & Arthritis & $\begin{array}{c}\text { Pyoderma } \\
\text { gangrenosum }\end{array}$ & $\begin{array}{l}\text { Erythema } \\
\text { nodosum }\end{array}$ & $\begin{array}{c}\text { Scleritis/ } \\
\text { Episcleritis }\end{array}$ & $\begin{array}{l}\text { Aphthous } \\
\text { ulcers }\end{array}$ & $\begin{array}{c}\text { Venous } \\
\text { thromboembolism }\end{array}$ \\
\hline Primary sclerosing cholangitis & - & $5.9(p=0.001)$ & $1.0(p=\mathrm{NS})$ & $0.6(0.53)$ & $0.5(p=0.46)$ & $1.0(p=\mathrm{NS})$ & $1.7(p=0.69)$ \\
\hline Arthritis & $5.9(p=0.001)$ & - & $1.6(p=0.34)$ & $3.1(p=0.008)$ & $2.0(p=0.08)$ & $2.1(p=0.03)$ & $5.0(p=0.01)$ \\
\hline Pyoderma gangrenosum & $1.0(p=\mathrm{NS})$ & $1.6(p=0.34)$ & - & $2.4(p=0.15)$ & $6.5(p<0.001)$ & $0.5(p=0.40)$ & $1.3(p=0.81)$ \\
\hline Erythema nodosum & $0.6(0.53)$ & $3.1(p=0.008)$ & $2.4(p=0.15)$ & - & $7.2(p<0.001)$ & $2.7(p=0.03)$ & $4.3(p=0.04)$ \\
\hline Scleritis/episcleritis & $0.5(p=0.46)$ & $2(p=0.08)$ & $6.5(p<0.001)$ & $7.2(p<0.001)$ & - & $2.3(p=0.07)$ & $0.9(p=0.92)$ \\
\hline Aphthous ulcers & $1.0(p=\mathrm{NS})$ & $2.1(p=0.03)$ & $0.5(p=0.40)$ & $2.7(p=0.03)$ & $2.3(p=0.07)$ & - & $2.4(p=0.60)$ \\
\hline Venous thromboembolism & $1.7(p=0.69)$ & $5.0(p=0.01)$ & $1.3(p=0.81)$ & $4.3(p=0.04)$ & $0.9(p=0.92)$ & $2.4(p=0.60)$ & - \\
\hline
\end{tabular}

Table 4 Simple and multiple regression analysis showing the association between EIM incidence and disease characteristics

\begin{tabular}{lcc}
\hline \multirow{2}{*}{$\begin{array}{l}\text { Extraintestinal manifestation } \\
\text { occurrence }\end{array}$} & $\begin{array}{c}\text { Odds ratio (95\% confidence } \\
\text { interval) }\end{array}$ \\
\cline { 2 - 3 } & Simple & Multiple \\
\hline $\begin{array}{l}\text { Inflammatory bowel disease subtype } \\
\quad \text { Crohn's disease/ulcerative colitis) }\end{array}$ & $0.91(0.56-1.47)$ & $\mathbf{0 . 5}(\mathbf{0 . 2 7}-\mathbf{0 . 0 3})$ \\
Gender (male/female) & $1.1(0.68-1.80)$ & $1.1(0.62-2.0)$ \\
Age at diagnosis & $\mathbf{1 . 0 3 ( 1 . 0 1 - 1 . 0 4 )}$ & $\mathbf{1 . 0 4}(\mathbf{1 . 0 1 - 1 . 0 6 )}$ \\
Smoking & $1.62(0.80-3.30)$ & $1.4(0.61-3.16)$ \\
Family history & $1.09(0.68-1.73)$ & $1.29(0.62-2.71)$ \\
Duration of disease & $1.02(0.96-1.09)$ & $0.99(0.26-1.18)$ \\
\hline
\end{tabular}

Bold indicates statistically significant.

with PSC $(p=0.001), \operatorname{EN}(p=0.008)$, aphthous ulcers $(p=0.03)$, and $\operatorname{VTE}(p=0.01)$. Ocular manifestations were also significantly associated with PG $(p<0.001)$ and $\mathrm{EN}(p<0.001)$. In addition, EN was associated with VTE $(p=0.04)$ and aphthous oral ulcers $(p=0.03)$, as shown in Table 3 .

\subsection{Multiple Regression Analysis}

On a multiple logistic regression analysis, EIM occurrence was associated with IBD subtype $(\mathrm{OR}=0.50, p=0.03)$ and age at the time of diagnosis $(\mathrm{OR}=1.04, p=0.001)$ (Table 4$)$.

On comparing individual EIMs with IBD subtype, there was a significant association between UC and PCS (OR $=26, p=0.004)$, and between $\mathrm{CD}$ and $\mathrm{PG}(\mathrm{OR}=0.22, p=0.03)$. EIM occurrence was predicted by age at diagnosis, as was the occurrence of arthritis (OR $=1.07, p<0.001)$, EN (OR $=1.04, p=0.02)$, scleritis/episcleritis $(\mathrm{OR}=1.04, p=0.009)$, and VTE $(\mathrm{OR}=1.1, p=0.001)$. Scleritis/ episcleritis occurrence had significant association with gender $(\mathrm{OR}=3.05, p=0.03)$, and VTE occurrence had significant association with duration of disease $(\mathrm{OR}=1.14, p=0.04)$.

\section{DISCUSSION}

The primary objective of this study was to estimate the incidence of EIMs in a Middle Eastern cohort of patients with IBD. Two hundred and eighty-four IBD patients' records were identified and reviewed using a database designed to follow up their clinical progress over 5 years. The EIMs investigated in our study included: EN; PG; aphthous ulcers; arthritis; PSC; scleritis and episcleritis; and VTEs. EIMs were encountered in almost half of the patients included in this population $(48.6 \%)$. This is considered a high percentage of EIMs compared with results from three large prospective cohort studies conducted in the USA, Switzerland, and India $[10,22,23]$, which reported incidence rates ranging between $36 \%$ and $38 \%$. The variation between incidence rates could be attributed to multiple factors such as differences in study design, inclusion and exclusion criteria, and geographical diversity. Other factors that may contribute to this variation include the differences between the diagnostic methods and definitions of EIMs between studies, and the completeness of patients' data during their follow up. According to hypothesis testing, no difference in the overall EIM incidence was observed between UC and CD patients ( 48.5 vs. 49.2, $p=0.70$ ), concordant with results of another cohort study in India [23]. However, multiple regression analysis showed a higher likelihood of developing EIMs in UC patients $(\mathrm{OR}=0.50, p=0.03)$.

The secondary objective of this study was to examine possible correlations between EIMs, and identify possible relationships between EIMs and other factors. There were indeed significant associations between IBD subtype and the occurrence of some EIMs. For exam- 
ple, arthritis and PSC were more common in UC patients, whereas PG was more common in CD patients. Nevertheless, arthritis was the most common EIM in this cohort, with a frequency of $33.3 \%$, consistent with a number of other studies [17,22,24-26]. As it is a diagnosis based primarily on clinical assessment, we considered any reported form of arthritis in this population as an EIM and did not classify it into type 1 and type 2 since we considered this classification to show no clinical value [27]. The high incidence of arthritis can be explained by the difficulty in differentiating between arthropathy and arthralgia. In addition, it could be a consequence of selected IBD medications, relapses, or extent of disease activity [22], as well as the malabsorptive nature of the disease [28]. According to the literature, certain genetic mutations are shared between IBD and rheumatic diseases, which might also explain the overlap in symptoms $[29,30]$. However, unlike previous studies, our study reveals that arthritis is significantly more common in UC $(p=0.02)[25,31,32]$.

The second most common EIM found in our study population was aphthous ulcers, which are considered part of the mucocutaneous manifestations of IBD that also include PG and EN. Aphthous ulcers, which frequently occur during IBD relapses $[2,4,14]$, are painful, round, pale-looking ulcers with a surrounding red halo [14,33]. Aphthous ulcers are diagnosed clinically and commonly occur with other diseases; they are sometimes labeled idiopathic [33], which might explain why it is the second most common EIM in our cohort with a frequency of $15.9 \%$. PG, which is considered the most severe skin manifestation of IBD, is an immune-mediated skin manifestation that arises more often in the lower extremities [14]. Similar to other studies, the overall incidence of PG in our center was not high (7.95\%) [34,35]. However, our report showed a significant association between PG and CD $(p=0.002)$, unlike previous studies that conversely linked PG with UC.

Finally, PSC, a chronic and progressive immuno-inflammatory disorder of the bile ducts [14], accounted for $6.8 \%$ of EIMs in our study and was significantly associated with UC $(p=0.001)$. PSC is generally difficult to diagnose owing to its vague clinical picture: patients are either asymptomatic or complain of nonspecific symptoms, and lab findings are inconclusive. Moreover, a definitive diagnosis can only be made by endoscopic retrograde cholangio pancreatography (ERCP) or magnetic resonance cholangio pancreatography (MRCP), which might explain its low incidence. PSC has been associated with a significant increase in the cumulative risk of colorectal cancer among UC patients [36]; thus, underscoring the importance of drawing attention to its diagnosis during the follow up of UC patients.

When evaluating the correlation of EIMs with each other, there was a significant association between arthritis and skin manifestations, namely $\operatorname{EN}(p=0.008)$ and aphthous ulcers $(p=0.03)$, arthritis and PSC $(p=0.001)$, and arthritis and VTE $(p=0.01)$. EN was also significantly associated with VTE $(p=0.04)$ and aphthous ulcers $(p=0.03)$. Furthermore, ocular manifestations were significantly associated with EN $(p=0.001)$ and PG $(p=0.001)$. Multiple studies have presented similar data to ours, finding a significant association between arthritis and skin manifestations, and the recurrence of aphthous ulcers in patients with other EIMs [31]. A 25-year follow-up study showed that ocular manifestations are more likely to be encountered with other EIMs [17], as did a Swiss cohort study, which reported associations between EIMs [22]. The most reasonable conclusion of the synchronicity of multiple EIMs is that all EIMs in this analysis are part of the overall inflammatory and immune-mediated process of IBD; some mimic each other in terms of pathogenesis and others have a similar inherited genetic component [30]. Thus, they tend to occur more during the active phase of IBD [17] and in conjunction with other EIMs. However, the multiplicity of factors in this investigation suggests that a clear cause may not be determined. The relevance of this reasoning is that although IBD is a disease of the gastrointestinal tract, other systems could be involved, and as multiple EIMs tend to coexist, the existence of one EIM can legitimately raise the suspicion of the presence of another. Thus, gastroenterologists should pay more attention to this by regularly assessing IBD patients with the engagement of other specialties to mitigate the overall risk of morbidity and mortality.

An important finding is that the only significant predictors of overall EIM incidence are IBD subtype and age at diagnosis. Multiple regression analysis demonstrated a higher probability of developing EIMs in UC patients $(\mathrm{OR}=0.50, p=0.03)$ and an increased EIM incidence with increased age at diagnosis of IBD $(\mathrm{OR}=1.04$, $p=0.001)$. However, this is discordant with some other studies, such as one conducted in Hungary that showed higher frequencies of EIMS in UC patients and a gender predisposition favoring females [17]. Disease subtype was also a significant predictor in the occurrence of two EIMs considered individually: PSC is more common in UC patients and PG more common in CD patients. Broadly, the existing literature reports similar data for the association between UC and PSC, but not for PG and CD.

In the present study, arthritis, EN, ocular manifestations, and VTE were all affected by age at diagnosis. Gender and disease duration were associated with the incidence of ocular manifestations and VTE, respectively. When following up IBD patients, predictors can be used as clinical tools that help to judge the likelihood of EIMs in different individuals with respect to the variation of disease characteristics between them. However, it remains a vague area in the literature and requires further research.

The limitations of this study supervene on the retrospective nature of data collection, which resulted in our data suffering from missing clinical information. Not all EIMs were accurately diagnosed as we relied on the clinical diagnosis mentioned in patients' files rather than diagnostic tests, which are needed to confirm certain EIMs, such as radiological investigations for PSC. The incidences of other EIMs, for example, osteopenia, nephrolithiasis, and uveitis, were not included. This also raises the concern of other confounding factors, which may have been not accounted for in analysis but which may have affected EIM occurrence, including comorbid diseases. Although the IBDIS registry includes only data from one center, it is continuously updated. This enabled us to have a thorough long-term follow up of patients. Another strength of the study is that we performed a multiple regression analysis to estimate EIM predictors, which enabled drawing more reliable associations.

\section{CONCLUSION}

Our results demonstrate that more than half of IBD patients have at least one EIM. Contrary to what has been reported generally, EIMs occur more commonly in UC compared with CD. IBD is an emerging health burden in our geographical area, thus an increased awareness of the possible implications of IBD in the form of EIMs has become a necessity in the Middle East to guide patients in seeking appropriate 
medical care. Also, this study has shown that the management of EIMs by other specialties should be done in close collaboration with gastroenterologists to effectively adjust the therapeutic regimens and to improve the morbidity and mortality of IBD patients.

\section{CONFLICTS OF INTEREST}

The authors declare they have no conflicts of interest.

\section{AUTHORS' CONTRIBUTION}

MM contributed in guarantor of the article. MM, MA, HA, and OS contributed in development of study concept and design. MM, MA, HA and OS contributed in acquisition, analysis, and interpretation of the data. MM contributed in statistical analysis. MM, MA, $\mathrm{HA}$ and $\mathrm{OS}$ contributed in drafting of the manuscript. MM, MA, $\mathrm{HA}$ and OS contributed in critical revision of the manuscript for important intellectual content.

\section{FUNDING}

This research project is eligible for the Saudi IBD master class grant once published.

\section{ACKNOWLEDGMENTS}

We would like to thank Dr. Trevor Rawbone, Cardiff, UK, for kindly reviewing and editing the manuscript.

\section{ABBREVIATIONS}

OR, odds ratio; CI, confidence interval; UC, ulcerative colitis; CD, Crohn's disease.

\section{ETHICAL APPROVAL AND CONSENT TO PARTICIPATE}

Ethical approval was obtained from King Abdulaziz University research ethics board.

\section{REFERENCES}

[1] Danese S, Sans M, Fiocchi C. Inflammatory bowel disease: the role of environmental factors. Autoimmun Rev 2004;3;394-400.

[2] Molodecky NA, Soon IS, Rabi DM, Ghali WA, Ferris M, Chernoff $\mathrm{G}$, et al. Increasing incidence and prevalence of the inflammatory bowel diseases with time, based on systematic review. Gastroenterology 2012;142;46.e42-54.e42;quiz e30.

[3] Isene R, Munkholm P, Stockbrügger R, Odes S, Bernklev T, Moum B. M1126 extraintestinal manifestations of inflammatory bowel disease and their impact on quality of life: results from the European Collaborative Study of Inflammatory Bowel Disease (EC-IBD). Gastroenterology 2009;136;A-355.
[4] Danese S, Semeraro S, Papa A, Roberto I, Scaldaferri F, Fedeli G, et al. Extraintestinal manifestations in inflammatory bowel disease. World J Gastroenterol 2005;11;7227-36.

[5] Bernstein CN, Blanchard JF, Rawsthorne P, Yu N. The prevalence of extraintestinal diseases in inflammatory bowel disease: a population-based study. Am J Gastroenterol 2001;96;1116-22.

[6] Ricart E, Panaccione R, Loftus EV, Tremaine WJ, Harmsen WS, Zinsmeister AR, et al. Autoimmune disorders and extraintestinal manifestations in first-degree familial and sporadic inflammatory bowel disease: a case-control study. Inflamm Bowel Dis 2004;10;207-14.

[7] Mendoza JL, Lana R, Taxonera C, Alba C, Izquierdo S, DíazRubio M. [Extraintestinal manifestations in inflammatory bowel disease: differences between Crohn's disease and ulcerative colitis]. Med Clin (Barc) 2005;125;297-300 [Article in Spanish].

[8] Rankin GB, Watts HD, Melnyk CS, Kelley ML. National Cooperative Crohn's Disease Study: extraintestinal manifestations and perianal complications. Gastroenterology 1979;77;914-20.

[9] Su CG, Judge TA, Lichtenstein GR. Extraintestinal manifestations of inflammatory bowel disease. Gastroenterol Clin North Am 2002;31;307-27.

[10] Greenstein AJ, Janowitz HD, Sachar DB. The extra-intestinal complications of Crohn's disease and ulcerative colitis: a study of 700 patients. Medicine (Baltimore) 1976;55;401-12.

[11] Farmer RG, Hawk WA, Turnbull RB. Clinical patterns in Crohn's disease: a statistical study of 615 cases. Gastroenterology 1975;68; 627-35.

[12] Wang YF, Ouyang Q, Hu RW. Progression of inflammatory bowel disease in China. J Dig Dis 2010;11;76-82.

[13] Wang YF, Zhang H, Ouyang Q. Clinical manifestations of inflammatory bowel disease: east and west differences. J Dig Dis $2007 ; 8 ; 121-7$.

[14] Larsen S, Bendtzen K, Nielsen OH. Extraintestinal manifestations of inflammatory bowel disease: epidemiology, diagnosis, and management. Ann Med 2010;42;97-114.

[15] Juillerat P, Mottet C, Pittet V, Froehlich F, Felley C, Gonvers JJ, et al. Extraintestinal manifestations of Crohn's disease. Digestion 2007;76;141-8.

[16] Bandyopadhyay D, Bandyopadhyay S, Ghosh P, De A, Bhattacharya A, Dhali GK, et al. Extraintestinal manifestations in inflammatory bowel disease: prevalence and predictors in Indian patients. Indian J Gastroenterol 2015;34;387-94.

[17] Lakatos L, Pandur T, David G, Balogh Z, Kuronya P, Tollas A, et al. Association of extraintestinal manifestations of inflammatory bowel disease in a province of western Hungary with disease phenotype: results of a 25-year follow-up study. World J Gastroenterol 2003;9;2300-7.

[18] Kethu SR. Extraintestinal manifestations of inflammatory bowel diseases. J Clin Gastroenterol 2006;40;467-75.

[19] Alharbi OR, Azzam NA, Almalki AS, Almadi MA, Alswat KA, Sadaf N, et al. Clinical epidemiology of ulcerative colitis in Arabs based on the Montréal classification. World J Gastroenterol 2014;20;17525-31.

[20] Aljebreen AM, Alharbi OR, Azzam NA, Almalki AS, Alswat KA, Almadi MA. Clinical epidemiology and phenotypic characteristics of Crohn's disease in the central region of Saudi Arabia. Saudi J Gastroenterol 2014;20;162-9.

[21] Alreheili KM, Alsaleem KA, Almehaidib AI. Natural history and outcome of inflammatory bowel diseases in children in Saudi Arabia: a single-center experience. Saudi J Gastroenterol 2018;24;171-6. 
[22] Vavricka SR, Brun L, Ballabeni P, Pittet V, Vavricka BMP, Zeitz J, et al. Frequency and risk factors for extraintestinal manifestations in the Swiss inflammatory bowel disease cohort. The Am J Gastroenterol 2011;106;110-19.

[23] Singh B, Kedia S, Konijeti G, Mouli VP, Dhingra R, Kurrey L, et al. Extraintestinal manifestations of inflammatory bowel disease and intestinal tuberculosis: frequency and relation with disease phenotype. Indian J Gastroenterol 2015;34;43-50.

[24] Meuwissen SG, Crusius BJ, Peña SA, Dekker-Saeys AJ, Dijkmans BA. Spondyloarthropathy and idiopathic inflammatory bowel diseases. Inflamm Bowel Dis 1997;3;25-37.

[25] Veloso FT. Inflammatory extraintestinal manifestations associated with IBD. In: Monteiro E, Tavarela Veloso F, editors. Inflammatory bowel diseases: new insight into mechanisms of inflammation and challenges in diagnosis and treatment. Lancaster: Kluwer Academic Publishers; 1995, pp. 232-6.

[26] Herfarth H, Obermeier F, Andus T, Rogler G, Nikolaus S, Kuehbacher $\mathrm{T}$, et al. Improvement of arthritis and arthralgia after treatment with infliximab (Remicade) in a German prospective, open-label, multicenter trial in refractory Crohn's disease. Am J Gastroenterol 2002;97;2688-90.

[27] Vavricka SR, Schoepfer A, Scharl M, Lakatos PL, Navarini A, Rogler G. Extraintestinal manifestations of inflammatory bowel disease. Inflamm Bowel Dis 2015;21;1982-92.

[28] Scarpa R, D’Arienzo A, Del Puente A, Panarese A, di Girolamo C, Larizza G, et al. Reverse correlation between extent of colon involvement and number of affected joints in patients with ulcerative colitis and arthritis. Am J Gastroenterol $1990 ; 85 ; 331-2$.

[29] Caillat-Zucman S. Molecular mechanisms of HLA association with autoimmune diseases. Tissue Antigens 2009;73;1-8.

[30] Turkcapar N, Toruner M, Soykan I, Aydintug OT, Cetinkaya H, Duzgun N, et al. The prevalence of extraintestinal manifestations and HLA association in patients with inflammatory bowel disease. Rheumatol Int 2006;26;663-8.

[31] Veloso FT, Carvalho J, Magro F. Immune-related systemic manifestations of inflammatory bowel disease: a prospective study of 792 patients. J Clin Gastroenterol 1996;23;29-34.

[32] Rothfuss KS, Stange EF, Herrlinger KR. Extraintestinal manifestations and complications in inflammatory bowel diseases. World J Gastroenterol 2006;12;4819-31.

[33] Ślebioda Z, Szponar E, Kowalska A. Etiopathogenesis of recurrent aphthous stomatitis and the role of immunologic aspects: literature review. Arch Immunol Ther Exp (Warsz) 2014;62;205-15.

[34] Yüksel İ, Başar Ö, Ataseven H, Ertuğrul İ, Arhan M, İbiş M, et al. Mucocutaneous manifestations in inflammatory bowel disease. Inflamm Bowel Dis 2009;15;546-50.

[35] Campbell S, Cripps S, Jewell DP. Therapy Insight: pyoderma gangrenosum-old disease, new management. Nat Clin Pract Gastroenterol Hepatol 2005;2;587-94.

[36] Florin TH, Pandeya N, Radford-Smith GL. Epidemiology of appendicectomy in primary sclerosing cholangitis and ulcerative colitis: its influence on the clinical behaviour of these diseases. Gut 2004;53;973-9. 